\title{
Spiritual Care - Modewort, Trend oder echte Notwendigkeit?
}

\author{
Die Beschäftigung mit den spirituellen Bedürfnissen der Patienten gehört nicht \\ zum üblichen ärztlichen Aufgabengebiet. Doch dieser Aspekt kann nicht einfach \\ auf die Klinikseelsorge abgeschoben werden. Patienten möchten mit ihrem Arzt \\ auch über existentielle und religiöse Fragen sprechen können.
}

René Heftia,

Stefan Rademacher ${ }^{b}$

Hans-RudolfPfeifer ${ }^{\text {, }}$ Rahel Gürber ${ }^{d}$

a Chefarzt und Ärztlicher Leiter Klinik SGM Langenthal; Dozent für Psychosoziale Medizin Universität Bern

b Wissenschaftlicher Mitarbeiter Forschungsinstitut für Spiritualität und Gesundheit (www.fisg.ch)

c Präsident Arbeitsgemeinschaft der evangelischen Ärztinnen und Ärzte der Schweiz

d Präsidentin der Vereinigung Katholischer Ärzte der Schweiz
Korrespondenz:

Dr. med. René Hefti

Klinik SGM Langenthal Weissensteinstrasse 30

CH-4900 Langenthal

Tel. 0629192211

Fax 0629192200

rene.hefti[at]klinik-sgm.ch
Die Schweizerische Ärztezeitung hat in den letzten Monaten mehrere Beiträge über «Medizin und Spiritualität» publiziert. Das Thema gewinnt in der Medizin an Bedeutung und wird kontrovers diskutiert. Soll die Ärztin oder der Arzt sich auch um die spirituellen Bedürfnisse der Patienten kümmern, oder ist das alleine Aufgabe der Seelsorge? Beeinflussen Religiosität und Spiritualität den Krankheits- und Heilungsverlauf und sind sie deshalb als Belastung oder Ressource in der medizinischen Behandlung zu berücksichtigen? Diesen Fragen wollen dieser Beitrag und eine gleichnamige Ärztetagung nachgehen.

\section{Spiritual Care - ein neues Behandlungsmodell}

Das Spiritual-Care-Konzept, das sich aus der Palliative Care heraus entwickelt hat, geht davon aus, dass Spiritualität eine relevante vierte Dimension im biopsychosozialen Modell darstellt $[1,2]$. Das interdisziplinäre Behandlungsteam hat die Aufgabe, sich um den ganzen Patienten zu kümmern («total patient care») und damit auch die spirituellen Bedürfnisse und Nöte zu integrieren [3, 4]. Die Klinikseelsorge ist Teil des interdisziplinären Teams, unterstützt dieses , ist aber nicht alleine zuständig für die spirituellen und religiösen Belange der Patienten. Sie übernimmt vielmehr die Rolle des «religiösen Experten», der wie die anderen Fachdisziplinen gezielt ihre Kompetenzen einbringt. Die Ärzte sind im Rahmen des Spiritual-Care-Modells gefordert, auch hinsichtlich der Spiritualität eine aktive Rolle zu übernehmen. Dies gilt insbesondere für das Erheben einer kurzen spirituellen Anamnese [5]. Dabei geht es um die Klärung der Frage, ob eine religiöse oder spirituelle Orientierung für den Patienten in der aktuellen Krankheitssituation eine bedeutsame Rolle spielt. Wichtig sind ein patientenzentriertes Vorgehen und ein offener Spiritualitätsbegriff [3, 4].

Dieses Spiritual-Care-Modell entspricht nicht dem aktuellen Selbstverständnis von uns Ärztinnen und Ärzten. Vielmehr wurden wir gelehrt, Wissenschaft und Religion strikte zu trennen. Die Beschäftigung mit den spirituellen Bedürfnissen unserer Patienten gehört nicht zu unserem üblichen Leis- tungskatalog. Vielmehr befürchten wir dabei eine Rollenkonfusion, den Verlust unserer professionellen ärztlichen Haltung und der damit verbundenen Neutralität. Zudem sind wir für spirituelle Belange nicht ausgebildet, obwohl wir für Patientinnen und Patienten in existentiellen Krankheitskrisen oft die ersten Ansprechpartner sind. Untersuchungen zeigen, dass Patientinnen und Patienten durchaus das Bedürfnis haben, mit ihren Ärztinnen und Ärzten existentielle und damit auch religiöse Fragen zu besprechen, da sie zu ihnen oft ein langjähriges Vertrauensverhältnis aufgebaut haben [6].

\section{Einfluss von Religiosität und Spiritualität}

An der Frage, ob Religiosität und Spiritualität auf Krankheits-, Bewältigungs- und Heilungsprozesse Einfluss haben, entscheidet sich ihre klinische Relevanz. Diese Frage wird im Rahmen der Religion and Health-Forschung in den USA (seit ca. 1980) und zunehmend auch in Europa empirisch untersucht. Hier einige Hinweise auf den aktuellen Kenntnisstand. Eine 2011 publizierte Metaanalyse [7] fasst 44 systematisch ausgewählte, prospektive Bevölkerungsstudien, welche den Zusammenhang zwischen Religiosität und Mortalität untersuchten, zusammen. Für die Bevölkerungsgruppen mit höherer Religiosität ergab sich eine Reduktion der Gesamtmortalität von $18 \%(\mathrm{HR}=0,82,95 \% \mathrm{CI}=0,76-0,87)$, was einem beachtlichen protektiven oder salutogenen Effekt der Religiosität entspricht. Für die kardiovaskuläre Mortalität betrug die Risikoreduktion sogar $28 \%(\mathrm{HR}=0,72,95 \% \mathrm{CI}=0,58-0,89)$. Interessanterweise profitierten die Frauen stärker als die Männer. Bei den Frauen betrug die Reduktion der Gesamtmortalität 30\%, während sie bei den Männern bei 13\% lag. Die Autoren erklären den günstigen Effekt der Religiosität auf die Mortalität durch besseres Gesundheitsverhalten, weniger Distress und gleichzeitig höhere Stressresistenz, mehr soziale Unterstützung, positive Emotionen und eine günstige Beeinflussung der autonomen Balance.

Ein weiterer wichtiger Aspekt von Religiosität und Spiritualität ist, wie eine Vielzahl von Studien zeigt [8], die Unterstützung in der Krankheitsbewäl- 


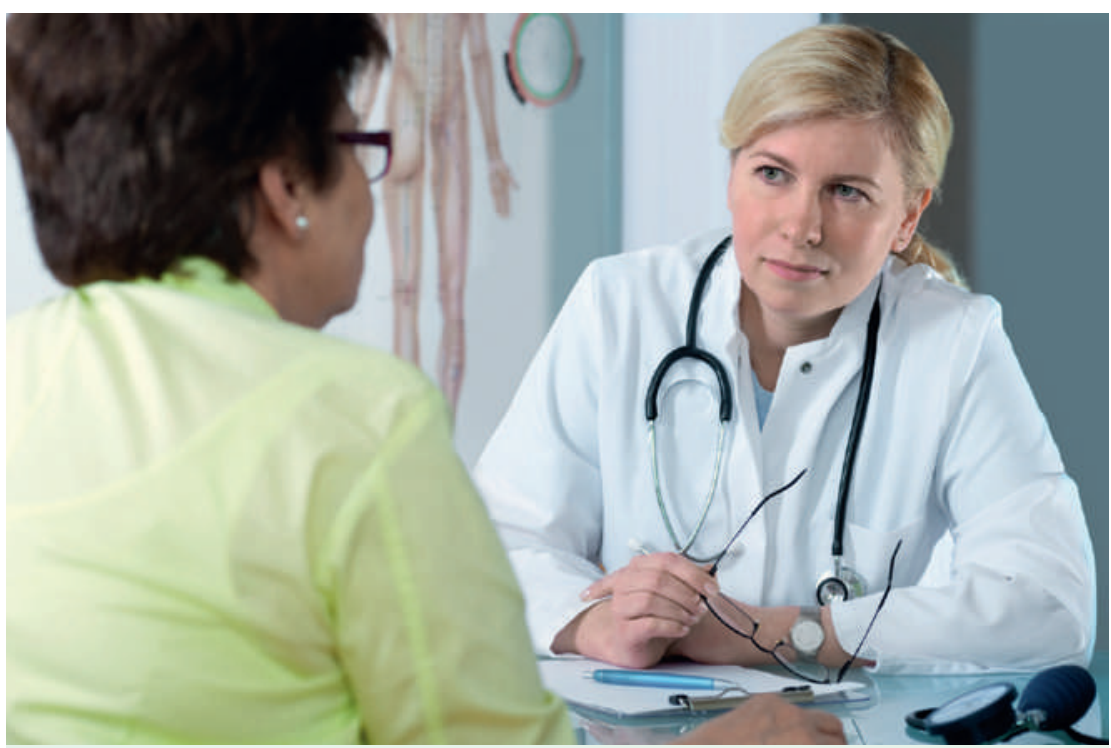

Viele Patienten haben das Bedürfnis, mit ihrem Arzt auch über existentielle und religiöse Fragen zu sprechen. Oft genügt es, wenn der Arzt, die Ärztin aufmerksam zuhört.

tigung. Man spricht von «religiösem oder spirituellem Coping» [9]. Religiosität und Spiritualität werden dabei als zusätzliche Ressourcen verstanden. Koenig untersuchte 337 Patienten [10], die auf die medizinische Abteilung des Duke University Hospitals aufgenommen werden mussten, und befragte sie hinsichtlich religiösem Coping. 67,4\% der Patientinnen und Patienten berichteten, dass Religiosität für sie ein wichtiger Faktor in der Bewältigung der aktuellen Krankheitssituation ist. In einer weiteren Untersuchung an 991 Patienten fanden sich für diejenigen, die Religiosität oder Spiritualität als Coping einsetzten, eine geringere Depressionsrate und damit eine erfolgreichere Krankheitsbewältigung.

Umgekehrt können Religiosität und Spiritualität im Krankheitsprozess auch zusätzliche Belastungsund Risikofaktoren darstellen. Eine prospektive Untersuchung an 596 medizinischen Patienten ergab für «Religious Struggles» eine Zunahme des Mortalitätsrisikos von 6-28\%, je nach Ausprägung und Art des religiösen Konfliktes [11]. Besonders ungünstig war, sich von Gott verlassen zu fühlen, an Gottes Liebe zu zweifeln oder die Krankheit dem Teufel zu attribuieren. Folgerichtig hat Stefanie Monod am CHUV in Lausanne ein «Spiritual Distress Assessment Tool» entwickelt [12], um solche Risikokonstellationen bei den Patienten zu erkennen und in den

Tagung «Spiritual Care - Modewort, Trend oder echte Notwendigkeit?»

Vom 9.-10. November 2013 findet in Quarten die gemeinsame Tagung der Arbeitsgemeinschaft Evangelischer Ärztinnen und Ärzte der Schweiz (AGEAS) und der Vereinigung Katholischer Ärzte der Schweiz (VKAS) statt. Informationen und Anmeldung finden sich unter www. ageas.ch oder www.medcath.ch
Behandlungsprozess einzubeziehen. Religiöse Zweifel oder Konflikte werden die Patienten dem Arzt selten spontan berichten, dazu braucht es ein gezieltes Nachfragen im Rahmen einer spirituellen Anamnese oder eines systematischen Assessments.

\section{Lehrstuhl für Spiritual Care?}

Um diesen Erkenntnissen Rechnung zu tragen, wurde an der Medizinischen Fakultät der LudwigMaximilians-Universität in München ein Lehrstuhl für Spiritual Care eingerichtet. Lehrstuhlinhaber sind Prof. Dr. med. Eckhard Frick und Prof. Dr. theol. Niels Hvidt, womit die medizinische wie auch die theologische Seite der Thematik abgedeckt ist. Ziel des Lehrstuhles ist es, Forschung im Bereich von Spiritual Care zu fördern und bereits die Medizinstudenten für diese Thematik zu sensibilisieren. Auch in Zürich wird nun auf Initiative der Kirchen ein solcher Lehrstuhl diskutiert

Der Beitrag wollte aufzeigen, dass die Diskussion über «Spiritual Care» in der Medizin relevant, aber noch lange nicht abgeschlossen ist. Es handelt sich mit Bestimmtheit um mehr als eine blosse Modeerscheinung oder einen aktuellen Trend. Dies zeigt auch die Zunahme der Beiträge zum Thema. Eine Ärztetagung in Quarten am 9./10. November 2013, an der Professor Frick als Hauptreferent teilnimmt, bietet Gelegenheit, sich mit der Thematik weiter auseinanderzusetzen.

\section{Literatur}

1 Hefti R. Spiritualität - die vierte Dimension oder der vergessene Faktor im biopsychosozialen Modell. Primary Care. 2010;(10):259-60.

2 Sulmasy DP. A biopsychosocial-spiritual model for the care of patients at the end of life. Gerontologist. 2002;42(3): 24-33.

3 Frick E. Spiritual Care: ein neues Fachgebiet der Medizin. Z Med Ethik. 2009; 55(2):145-5.

4 Koenig HA. Spiritualität in den Gesundheitsberufen. Ein praxisorientierter Leitfaden. Stuttgart: Kohlhammer; 2012.

5 Puchalski CM, Romer AL. Taking a Spiritual History Allows Clinicians to Understand Patients More Fully. Journal of Palliative Medicine. 2000(3):129-37.

6 Daaleman TP, Nease DE. Patient attitudes regarding physician inquiry into spiritual and religious issues. Journal of Family Practice. 1994;(39):564-8.

7 Chida Y, Steptow A, Powell LH. Religiosity/spirituality and mortality. Psychotherapy and Psychosomatics. 2009; (78):81-90.

8 Koenig HG, King DE, Carson VB. Handbook of religion and health, second edition. New York: Oxford University Press; 2012.

9 Pargament K. The psychology of religion and coping: Theory, research, and practice. New York: Guilford Press; 1997.

10 Koenig HG. Religious beliefs and practices of hospitalized medically ill older adults. International Journal of Geriatric Psychiatry. 1998;(13):213-24.

11 Pargament, KI., Koenig HG, Tarakeshwar N, Hahn J. Religious struggle as a predictor of mortality among medically ill elderly patients. Archive of Internal Medicine. 2001;(161):1881-5.

12 Monod SM, Rochat E, Bula CJ, Jobin G, Martin E, Spencer B. The Spiritual Distress Assessment Tool: An instrument to assess spiritual distress in hospitalised elderly; 2010. 